\title{
On Estimates of Deviation of Functions from Matrix Operators of Their Fourier Series by Some Expressions with $r$-Differences of the Entries
}

\section{Włodzimierz Lenski and Bogdan Szal}

Faculty of Mathematics, Computer Science and Econometrics, University of Zielona Góra, Ulica Szafrana 4a, 65-516 Zielona Góra, Poland

Correspondence should be addressed to Bogdan Szal; b.szal@wmie.uz.zgora.pl

Received 15 October 2015; Revised 11 February 2016; Accepted 23 March 2016

Academic Editor: Sergey Tikhonov

Copyright (C) 2016 W. Łenski and B. Szal. This is an open access article distributed under the Creative Commons Attribution License, which permits unrestricted use, distribution, and reproduction in any medium, provided the original work is properly cited.

We generalize the results of Krasniqi 2012 and Wei and Yu 2012 to the case of $r$-differences.

\section{Introduction}

Let $X=L^{p}$ or $X=C$, where $L^{p}(1 \leq p \leq \infty)[C]$ is the class of all $2 \pi$-periodic real-valued functions, integrable in the Lebesgue sense with $p$ th power when $p \geq 1$ and essentially bounded when $p=\infty$ [continuous], over $Q=[-\pi, \pi]$ with the norm

$$
\begin{aligned}
\|f\|_{L^{p}} & :=\|f(\cdot)\|_{L^{p}} \\
& = \begin{cases}\left(\int_{Q}|f(t)|^{p} d t\right)^{1 / p} & \text { when } 1 \leq p<\infty, \\
\operatorname{ess} \sup _{t \in Q}|f(t)| & \text { when } p=\infty,\end{cases} \\
\|f\|_{C}: & =\|f(\cdot)\|_{C}=\sup _{t \in Q}|f(t)|
\end{aligned}
$$

and consider the trigonometric Fourier series

$$
S f(x):=\frac{a_{0}(f)}{2}+\sum_{\nu=1}^{\infty}\left(a_{\nu}(f) \cos v x+b_{\nu}(f) \sin v x\right)
$$

with the partial sums $S_{k} f$.

Let $A:=\left(a_{n, k}\right)$ be an infinite matrix of real numbers such that

$$
a_{n, k} \geq 0
$$

$$
\text { when } k, n=0,1,2, \ldots, \lim _{n \rightarrow \infty} a_{n, k}=0, \sum_{k=0}^{\infty} a_{n, k}=1 .
$$

The A-transformation of $\left(S_{k} f\right)$ be given by

$$
T_{n, A} f(x):=\sum_{k=0}^{\infty} a_{n, k} S_{k} f(x) \quad(n=0,1,2, \ldots) .
$$

In this paper, we study the upper bounds of $\left\|T_{n, A} f-f\right\|_{X}$ by the second modulus of continuity of $f$ in the space $X$ defined by the formula

$$
\omega_{2}(f, \delta)_{X}:=\sup _{0 \leq|t| \leq \delta}\left\|\varphi_{x}(t)\right\|_{X},
$$

where

$$
\varphi_{x}(t):=f(x+t)+f(x-t)-2 f(x) .
$$

The deviation $T_{n, A} f-f$ with lower triangular infinite matrix such that

$$
\begin{array}{rlrl}
a_{n, k} \geq 0 & & \text { when } k=0,1,2, \ldots, n, \\
a_{n, k} & =0 & & \text { when } k>n, \\
\sum_{k=0}^{n} a_{n, k} & =1, & & \text { where } n=0,1,2, \ldots,
\end{array}
$$

was estimated in the sup norm $\|\cdot\|_{C}$ by Krasniqi [1, Theorem 3.4, p. 290] (see also [2]) as follows. 
Theorem 1. Let $f \in C$ and $\left(a_{n, k}\right)$ satisfy (8). Then,

$$
\begin{aligned}
& \left\|T_{n, A} f-f\right\|_{C}=O\left(\omega\left(f, \frac{\pi}{n}\right)_{C}\right. \\
& +\sum_{k=1}^{n} \frac{\omega(f, \pi / k)_{C}}{k} \sum_{l=0}^{k+1} a_{n, l} \\
& \left.+\sum_{k=1}^{n} \omega\left(f, \frac{\pi}{k}\right)_{C} \sum_{l=k}^{n}\left|a_{n, l}-a_{n, l+2}\right|\right),
\end{aligned}
$$

where $\omega(f, \cdot)_{C}$ denotes the modulus of continuity of $f$ and if $\omega(f, \cdot)_{C}$ is such that

$$
\int_{u}^{\pi} t^{-2} \omega(f, t)_{C} d t=O(H(u)) \quad \text { when } u \in[0, \pi],
$$

where $H(u) \geq 0$, we have

$$
\begin{aligned}
& \left\|T_{n, A} f-f\right\|_{C} \\
& \quad=O\left(\omega\left(f, \frac{\pi}{n}\right)_{C}+H\left(\frac{\pi}{n}\right) \sum_{k=0}^{n}\left|a_{n, k}-a_{n, k+2}\right|\right) .
\end{aligned}
$$

Additionally, if $H$ satisfies the condition

$$
\int_{0}^{u} H(t) d t=O(u H(u)) \quad \text { when } u \in[0, \pi]
$$

then

$$
\begin{aligned}
& \left\|T_{n, A} f-f\right\|_{C} \\
& =O\left(H\left(\sum_{k=0}^{n}\left|a_{n, k}-a_{n, k+2}\right|\right) \sum_{k=0}^{n}\left|a_{n, k}-a_{n, k+2}\right|\right), \\
& \left\|T_{n, A} f-f\right\|_{C}=O\left(H\left(\frac{\pi}{n}\right) \sum_{k=0}^{n}\left|a_{n, k}-a_{n, k+2}\right|\right) .
\end{aligned}
$$

In our theorems we will consider the $r$-differences $a_{n, k}-$ $a_{n, k+r}$ instead of $a_{n, k}-a_{n, k+2}$ considered above. We will formulate the general relation for $r \in \mathbb{N}$ like it formulated only for $r=2$ in [1, Theorem 3.4, p. 290].

\section{Statement of the Results}

Let us consider a function $\omega$ of modulus of continuity type on the interval $[0,2 \pi]$, that is, a nondecreasing continuous function having the following properties: $\omega(0)=0, \omega\left(\delta_{1}+\right.$ $\left.\delta_{2}\right) \leq \omega\left(\delta_{1}\right)+\omega\left(\delta_{2}\right)$ for any $0 \leq \delta_{1} \leq \delta_{2} \leq \delta_{1}+\delta_{2} \leq 2 \pi$.

Suppose that $\omega$ satisfies the condition

$$
\int_{u}^{\pi} t^{-2} \omega(t) d t=O(H(u)) \quad \text { when } u \in[0, \pi],
$$

where $H(u) \geq 0$ is such that

$$
\int_{0}^{u} H(t) d t=O(u H(u)) \quad \text { when } u \in[0, \pi] .
$$

Let

$$
\begin{aligned}
X_{\omega} & =\left\{f \in X: \omega_{2}(f, \delta)_{X}=O(\omega(\delta)) \text { when } \delta\right. \\
& \in[0,2 \pi]\} .
\end{aligned}
$$

Our main results on the degrees of approximation are the following.

Theorem 2. If $f \in X_{\omega}$, where $\omega$ satisfies condition (14) such that (15) holds and $r \in \mathbb{N}$, then

$$
\begin{aligned}
& \left\|T_{n, A} f-f\right\|_{X} \\
& \quad=O\left(H\left(\frac{\pi}{n+1}\right)\left(\frac{\pi}{n+1}+\sum_{k=0}^{\infty}\left|a_{n, k}-a_{n, k+r}\right|\right)\right) .
\end{aligned}
$$

Additionally, if a matrix $A$ is such that

$$
\left[\sum_{l=0}^{n} \sum_{k=l}^{r+l-1} a_{n, k}\right]^{-1}=O(1)
$$

then

$$
\left\|T_{n, A} f-f\right\|_{X}=O\left(H\left(\frac{\pi}{n+1}\right) \sum_{k=0}^{\infty}\left|a_{n, k}-a_{n, k+r}\right|\right) .
$$

Theorem 3. If $f \in X_{\omega}$, where $\omega$ satisfies condition (14) such that (15) holds and $r \in \mathbb{N}$, then

$$
\begin{aligned}
& \left\|T_{n, A} f-f\right\|_{X} \\
& \quad=O\left(H\left(\sum_{k=0}^{\infty}\left|a_{n, k}-a_{n, k+r}\right|\right) \sum_{k=0}^{\infty}\left|a_{n, \mathrm{k}}-a_{n, k+r}\right|\right) .
\end{aligned}
$$

Theorem 4. Let $f \in X_{\omega}$, where $\omega$ satisfies condition (14) such that (15) holds and $r \in \mathbb{N}$. If a matrix $A$ is such that

$$
\sum_{k=0}^{\infty}(k+1) a_{n, k}=O(n+1),
$$

then

$$
\begin{aligned}
& \left\|T_{n, A} f-f\right\|_{X} \\
& \quad=O\left(\omega\left(\frac{\pi}{n+1}\right)+H\left(\frac{\pi}{n+1}\right) \sum_{k=0}^{\infty}\left|a_{n, k}-a_{n, k+r}\right|\right) .
\end{aligned}
$$

Theorem 5. If $f \in X$ and a matrix $A$ is such that (21) holds and $r \in \mathbb{N}$, then

$$
\begin{aligned}
& \left\|T_{n, A} f-f\right\|_{X}=O\left(\omega_{2}\left(f, \frac{\pi}{n+1}\right)_{X}\right. \\
& +\sum_{\mu=1}^{n} \frac{\omega_{2}(f, \pi / \mu)_{X}}{\mu} \sum_{k=0}^{\mu+1} a_{n, k} \\
& \left.+\sum_{\mu=1}^{n} \omega_{2}\left(f, \frac{\pi}{\mu}\right)_{X} \sum_{k=\mu}^{\infty}\left|a_{n, k}-a_{n, k+r}\right|\right) .
\end{aligned}
$$


Corollary 6. For a lower triangular infinite matrix A conditions (18) and (21) hold always and therefore for a lower triangular infinite matrix such that conditions (7) and (8) hold one can obtain the results from Theorems 2, 4, and 5 without the assumptions (18) and (21), where the mentioned results of Krasniqi follow as the special cases with $r=2$. Moreover, one can consider the essentially wider class of sequences than in the mentioned paper with the same degrees of approximation (see, e.g., [3, Theorem 2]).

Corollary 7. From Theorem 5 it follows that if $f \in X_{\omega}$ where $\omega$ satisfies condition (14) such that (15) holds and a matrix $A$ is such that (21) and

$$
\sum_{k=m}^{\infty}\left|a_{n, k}-a_{n, k+r}\right| \leq K \sum_{k=m / c}^{\infty} \frac{a_{n, k}}{k},
$$

with some $c>1$ and $r \in \mathbb{N}$, hold, then

$$
\begin{aligned}
& \left\|T_{n, A} f-f\right\|_{X} \\
& \quad=O\left(\frac{H(\pi /(n+1))}{n+1}+\sum_{k=1}^{n} a_{n, k} \frac{H(\pi /(k+1))}{k+1}\right) .
\end{aligned}
$$

Remark 8. The class of sequences defined by condition (24) was introduced by the second author in [4]. The similar classes were considered by Dyachenko and Tikhonov in [5] with $r=1$ (see also [6]).

Remark 9. We note that instead of (14) and (15) one can use Bari-Stechkin conditions

$$
\begin{aligned}
& \int_{0}^{u} t^{-1} \omega(t) d t=O(\omega(u)), \\
& \int_{u}^{2 \pi} t^{-2} \omega(t) d t=O(\omega(u))
\end{aligned}
$$

when $u \in[0,2 \pi]$.

Then all results are true for $t^{-1} \omega(t)$ instead of $H(y)$ and Lemmas 12 and 14 are not necessary.

\section{Auxiliary Results}

We begin this section by some notations from [7]. Let for $r=$ $1,2, \ldots$

$$
\begin{aligned}
& D_{k, r}(t)=\frac{\sin ((2 k+r) t / 2)}{2 \sin (r t / 2)}, \\
& \widetilde{D}_{k, r}(t)=\frac{\cos ((2 k+r) t / 2)}{2 \sin (r t / 2)} .
\end{aligned}
$$

It is clear by [8] that

$$
\begin{aligned}
S_{k} f(x) & =\frac{1}{\pi} \int_{-\pi}^{\pi} f(x+t) D_{k, 1}(t) d t, \\
T_{n, A} f(x) & =\frac{1}{\pi} \int_{-\pi}^{\pi} f(x+t) \sum_{k=0}^{n} a_{n, k} D_{k, 1}(t) d t .
\end{aligned}
$$

Hence

$$
T_{n, A} f(x)-f(x)=\frac{1}{\pi} \int_{0}^{\pi} \varphi_{x}(t) \sum_{k=0}^{n} a_{n, k} D_{k, 1}(t) d t .
$$

Next, we present the known estimates.

Lemma 10 (see [8]). If $0<|t| \leq \pi$ then

$$
\begin{aligned}
& \left|D_{k, 1}(t)\right| \leq \frac{\pi}{2|t|}, \\
& \left|\widetilde{D}_{k, 1}(t)\right| \leq \frac{\pi}{2|t|}
\end{aligned}
$$

and, for any real $t$, we have

$$
\left|D_{k, 1}(t)\right| \leq k+\frac{1}{2} \text {. }
$$

Lemma 11 (see [7]). Let $r \in N, l \in Z$, and $a:=\left(a_{n}\right) \subset \mathbb{C}$. If $x \neq 2 l \pi / r$ then for every $m \geq n$

$$
\begin{aligned}
\sum_{k=n}^{m} a_{k} \sin k x= & -\sum_{k=n}^{m}\left(a_{k}-a_{k+r}\right) \widetilde{D}_{k, r}(t) \\
& +\sum_{k=m+1}^{m+r} a_{k} \widetilde{D}_{k,-r}(t) \\
& -\sum_{k=n}^{n+r-1} a_{k} \widetilde{D}_{k,-r}(t), \\
\sum_{k=n}^{m} a_{k} \cos k x= & \sum_{k=n}^{m}\left(a_{k}-a_{k+r}\right) D_{k, r}(t) \\
& -\sum_{k=m+1}^{m+r} a_{k} D_{k,-r}(t) \\
& +\sum_{k=n}^{n+r-1} a_{k} D_{k,-r}(t) .
\end{aligned}
$$

Lemma 12 (see [9]). If (14) and (15) hold then

$$
\int_{0}^{u} t^{-1} \omega(t) d t=O(u H(u)) \quad \text { when } u \in[0,2 \pi] .
$$

We additionally proved two slight changed estimates.

Lemma 13. If (14) and (15) hold, then, for $c \geq 1$ and $\beta>\alpha>0$,

$$
\begin{aligned}
& \int_{\alpha}^{\beta} t^{-1} \omega(t) d t=O((\beta-\alpha) H(c(\beta-\alpha))) \\
& \text { when }(\beta-\alpha) \in[0,2 \pi] .
\end{aligned}
$$

Proof. By substitution of $t=u+\alpha$ and monotonicity of $\omega(u) / u$ (see Lemma 15) we obtain, for $c \geq 1$,

$$
\begin{aligned}
\int_{\alpha}^{\beta} t^{-1} \omega(t) d t & =\int_{0}^{\beta-\alpha} \frac{\omega(u+\alpha)}{u+\alpha} d u \leq 2 \int_{0}^{\beta-\alpha} \frac{\omega(u)}{u} d u \\
& \leq 2 \int_{0}^{c(\beta-\alpha)} \frac{\omega(u)}{u} d u
\end{aligned}
$$

and the desired result follows from the Lemma 12. 
Lemma 14. If (14) and (15) hold, then, for $b \geq 1$,

$$
\int_{u}^{\pi} t^{-2} \omega(t) d t=O(H(b u)) \quad \text { when } u \in[0, \pi] .
$$

Proof. Using Lemma 13 with $c=1, \alpha=0$ and (14) our result follows

$$
\begin{aligned}
\int_{u}^{\pi} t^{-2} \omega(t) d t & =\left(\int_{u}^{b u}+\int_{b u}^{\pi}\right) t^{-2} \omega(t) d t \\
& \leq \frac{1}{u} \int_{u}^{b u} \frac{\omega(t)}{t} d t+O(H(b u)) \\
& \leq \frac{1}{u} \int_{0}^{b u} \frac{\omega(t)}{t} d t+O(H(b u)) \\
& =\frac{1}{u} O(b u H(b u))+O(H(b u)) \\
& =O((b+1) H(b u)),
\end{aligned}
$$

for $b \geq 1$.

Finally, we present very useful property of the modulus of continuity.

Lemma 15 (see [8]). A function $\omega$ of modulus of continuity type on the interval $[0,2 \pi]$ satisfies the following condition:

$$
\delta_{2}^{-1} \omega\left(\delta_{2}\right) \leq 2 \delta_{1}^{-1} \omega\left(\delta_{1}\right) \quad \text { for } \delta_{2} \geq \delta_{1}>0 .
$$

\section{Proofs of the Results}

Proof of Theorem 2. It is clear that for an odd $r$

$$
\begin{array}{rl}
T_{n, A} & f(x)-f(x) \\
= & \frac{1}{\pi} \sum_{m=0}^{[r / 2]} \int_{2 m \pi / r}^{2 m \pi / r+\pi / r} \varphi_{x}(t) \sum_{k=0}^{\infty} a_{n, k} D_{k, 1}(t) d t \\
& +\frac{1}{\pi} \sum_{m=0}^{[r / 2]-1} \int_{2 m \pi / r+\pi / r}^{2(m+1) \pi / r} \varphi_{x}(t) \sum_{k=0}^{\infty} a_{n, k} D_{k, 1}(t) d t \\
= & I_{1}(x)+I_{2}(x)
\end{array}
$$

and for an even $r$

$$
\begin{gathered}
T_{n, A} f(x)-f(x)=\frac{1}{\pi} \\
\cdot \sum_{m=0}^{[r / 2]-1}\left(\int_{2 m \pi / r}^{2 m \pi / r+\pi / r}+\int_{2 m \pi / r+\pi / r}^{2(m+1) \pi / r}\right) \varphi_{x}(t) \\
\cdot \sum_{k=0}^{\infty} a_{n, k} D_{k, 1}(t) d t=I_{1}^{\prime}(x)+I_{2}(x) .
\end{gathered}
$$

Then,

$$
\left\|T_{n, A} f-f\right\|_{X} \leq\left\|I_{1}\right\|_{X}+\left\|I_{1}^{\prime}\right\|_{X}+\left\|I_{2}\right\|_{X} .
$$

Since, by Lemma 11,

$$
\begin{aligned}
& \sum_{k=0}^{\infty} a_{n, k} D_{k, 1}(t)=\sum_{k=0}^{\infty} a_{n, k} \frac{\sin ((2 k+1) t / 2)}{2 \sin (t / 2)} \\
& \quad=\frac{1}{2 \sin (t / 2)}\left(\sum_{k=0}^{\infty} a_{n, k} \sin k t \cos (t / 2)\right. \\
& \left.\quad+\sum_{k=0}^{\infty} a_{n, k} \cos k t \sin (t / 2)\right) \\
& =\frac{\cos (t / 2)}{2 \sin (t / 2)}\left(-\sum_{k=0}^{\infty}\left(a_{n, k}-a_{n, k+r}\right) \widetilde{D}_{k, r}(t)\right. \\
& \left.\quad-\sum_{k=0}^{r-1} a_{n, k} \widetilde{D}_{k,-r}(t)\right)+\frac{1}{2}\left(\sum_{k=0}^{\infty}\left(a_{n, k}-a_{n, k+r}\right) D_{k, r}(t)\right. \\
& \left.\quad+\sum_{k=0}^{r-1} a_{n, k} D_{k,-r}(t)\right),
\end{aligned}
$$

then

$$
\begin{aligned}
& \left|\sum_{k=0}^{\infty} a_{n, k} D_{k, 1}(t)\right| \\
& \quad \leq \frac{1}{2|\sin (t / 2) \sin (r t / 2)|}\left(\sum_{k=0}^{\infty}\left|a_{n, k}-a_{n, k+r}\right|\right. \\
& \left.\quad+\sum_{k=0}^{r-1} a_{n, k}\right) \leq \frac{1}{|\sin (t / 2) \sin (r t / 2)|} \sum_{k=0}^{\infty}\left|a_{n, k}-a_{n, k+r}\right| .
\end{aligned}
$$

Hence, by Lemma 10,

$$
\begin{aligned}
& \left\|I_{1}\right\|_{X} \leq \frac{1}{\pi} \sum_{m=0}^{[r / 2]} \int_{2 m \pi / r}^{2 m \pi / r+\pi / r}\left\|\varphi_{x}(t)\right\|_{X}\left|\sum_{k=0}^{\infty} a_{n, k} D_{k, 1}(t)\right| d t=\frac{1}{\pi} \\
& \cdot \sum_{m=0}^{[r / 2]}\left(\int_{2 m \pi / r}^{2 m \pi / r+\pi / r(n+1)}+\int_{2 m \pi / r+\pi / r(n+1)}^{2 m \pi / r+\pi / r}\right)\left\|\varphi_{x}(t)\right\|_{X} \\
& \cdot\left|\sum_{k=0}^{\infty} a_{n, k} D_{k, 1}(t)\right| d t \leq \frac{1}{2} \sum_{m=0}^{[r / 2]} \int_{2 m \pi / r}^{2 m \pi / r+\pi / r(n+1)} \frac{O(\omega(t))}{t} d t \\
& +\frac{1}{\pi} \\
& \quad \cdot \sum_{m=0}^{[r / 2]} \int_{2 m \pi / r+\pi / r(n+1)}^{2 m \pi / r+\pi / r} \frac{O(\omega(t))}{|\sin (t / 2) \sin (r t / 2)|} \sum_{k=0}^{\infty}\left|a_{n, k}-a_{n, k+r}\right| d t .
\end{aligned}
$$

Using Lemmas 13 and 14 with $c=b=r$ and the estimates $|\sin (t / 2)| \geq|t| / \pi$ for $t \in[0, \pi]$ and $|\sin (r t / 2)| \geq r t / \pi-2 m$ for $t \in[2 m \pi / r, 2 m \pi / r+\pi / r]$, where $m \in\{0, \ldots,[r / 2]\}$, we obtain

$$
\left\|I_{1}\right\|_{X} \leq O(1)\left(\left[\frac{r}{2}\right]+1\right) \frac{\pi}{r(n+1)} H\left(\frac{\pi}{n+1}\right)
$$

$$
+\sum_{k=0}^{\infty}\left|a_{n, k}-a_{n, k+r}\right|
$$




$$
\begin{aligned}
& \cdot \sum_{m=0}^{[r / 2]} \int_{2 m \pi / r+\pi / r(n+1)}^{2 m \pi / r+\pi / r} \frac{O(\omega(t))}{t(r t / \pi-2 m)} d t=O(1) \frac{\pi}{n+1} \\
& \cdot H\left(\frac{\pi}{n+1}\right)+\sum_{k=0}^{\infty}\left|a_{n, k}-a_{n, k+r}\right| \\
& \cdot \sum_{m=0}^{[r / 2]} \int_{2 m \pi / r+\pi / r(n+1)}^{2 m \pi / r+\pi / r} \frac{O(\omega(t))}{(r t / \pi)(t-2 m \pi / r)} d t=O(1) \\
& \cdot \frac{\pi}{n+1} H\left(\frac{\pi}{n+1}\right)+\sum_{k=0}^{\infty}\left|a_{n, k}-a_{n, k+r}\right| \\
& \cdot \sum_{m=0}^{[r / 2]} \int_{\pi / r(n+1)}^{\pi / r} \frac{O(\omega t / \pi)(t+2 m \pi / r)}{(r t+2 m \pi / r))} d t \leq O(1) \\
& \cdot\left[\frac{\pi}{n+1} H\left(\frac{\pi}{n+1}\right)+\left(\left[\frac{r}{2}\right]+1\right) \frac{2 \pi}{r}\right. \\
& \left.+\sum_{k=0}^{\infty}\left|a_{n, k}-a_{n, k+r}\right| H\left(\frac{\pi}{n+1}\right)\right] \cdot \\
& \left.\left.\cdot \sum_{k=0}^{\infty}\left|a_{n, k}-a_{n, k+r}\right|\right]_{\pi / r(n+1)}^{\pi / r} \frac{\omega(t)}{t^{2}} d t\right]=O(1) \\
& \cdot\left[\frac{\pi}{n+1} H\left(\frac{\pi}{n+1}\right)\right. \\
& {\left[\begin{array}{c}
n+1 \\
n+1
\end{array}\right]}
\end{aligned}
$$

Analogously

$$
\begin{gathered}
\left\|I_{1}^{\prime}\right\|_{X}=O(1)\left[\frac{r}{2}\right]\left[\frac{\pi}{r(n+1)} H\left(\frac{\pi}{n+1}\right)\right. \\
\left.+\frac{2 \pi}{r} \sum_{k=0}^{\infty}\left|a_{n, k}-a_{n, k+r}\right| H\left(\frac{\pi}{n+1}\right)\right] .
\end{gathered}
$$

Similarly, by Lemmas 10,13 , and 14 with $c=b=r$ and the estimates $|\sin (t / 2)| \geq|t| / \pi$ for $t \in[0, \pi]$ and $|\sin (r t / 2)| \geq$ $2(m+1)-r t / \pi$ for $t \in[2(m+1) \pi / r-\pi / r, 2(m+1) \pi / r-$ $\pi / r(n+1)]$, where $m \in\{0, \ldots,[r / 2]-1\}$, we get

$$
\begin{array}{r}
\left\|I_{2}\right\|_{X} \leq \frac{1}{\pi} \sum_{m=0}^{[r / 2]-1} \int_{2 m \pi / r+\pi / r}^{2(m+1) \pi / r}\left\|\varphi_{x}(t)\right\|_{X}\left|\sum_{k=0}^{\infty} a_{n, k} D_{k, 1}(t)\right| d t \\
=\frac{1}{\pi} \sum_{m=0}^{[r / 2]-1}\left(\int_{2(m+1) \pi / r-\pi / r}^{2(m+1) \pi / r-\pi / r(n+1)}+\int_{2(m+1) \pi / r-\pi / r(n+1)}^{2(m+1) \pi / r}\right) \\
\cdot\left\|\varphi_{x}(t)\right\|_{X}\left|\sum_{k=0}^{\infty} a_{n, k} D_{k, 1}(t)\right| d t \leq \frac{1}{2} \\
\cdot \sum_{m=0}^{[r / 2]-1} \int_{2(m+1) \pi / r-\pi / r(n+1)}^{2(m+1) \pi / r} \frac{O(\omega(t))}{t} d t+\frac{1}{\pi} \sum_{k=0}^{\infty} \mid a_{n, k}
\end{array}
$$

$$
\begin{aligned}
& -a_{n, k+r} \\
& \cdot \sum_{m=0}^{[r / 2]-1} \int_{2(m+1) \pi / r-\pi / r}^{2(m+1) \pi / r-\pi / r(n+1)} \frac{O(\omega(t))}{|\sin (t / 2) \sin (r t / 2)|} d t \leq \frac{1}{2} \\
& \cdot \sum_{m=0}^{[r / 2]-1} \int_{2(m+1) \pi / r-\pi / r(n+1)}^{2(m+1) \pi / r} \frac{O(\omega(t))}{t} d t+\sum_{k=0}^{\infty} \mid a_{n, k} \\
& -a_{n, k+r} \\
& \cdot \sum_{m=0}^{[r / 2]-1} \int_{2(m+1) \pi / r-\pi / r}^{2(m+1) \pi / r-\pi / r(n+1)} \frac{O(\omega(t))}{(r t / \pi)[2(m+1) \pi / r-t]} d t \\
& =\frac{1}{2} \sum_{m=0}^{[r / 2]-1} \int_{2(m+1) \pi / r-\pi / r(n+1)}^{2(m+1) \pi / r} \frac{O(\omega(t))}{t} d t+\sum_{k=0}^{\infty} \mid a_{n, k} \\
& -a_{n, k+r} \mid \sum_{m=0}^{[r / 2]-1} \int_{\pi / r(n+1)}^{\pi / r} \frac{O(\omega(-t+2(m+1) \pi / r))}{(r / \pi) t(-t+2(m+1) \pi / r)} d t \\
& \leq \frac{1}{2} \sum_{m=0}^{[r / 2]-1} \int_{2(m+1) \pi / r-\pi / r(n+1)}^{2(m+1) \pi / r} \frac{O(\omega(t))}{t} d t+\sum_{k=0}^{\infty} \mid a_{n, k} \\
& -a_{n, k+r} \mid\left[\frac{r}{2}\right] \frac{2 \pi}{r} \int_{\pi / r(n+1)}^{\pi / r} \frac{O(\omega(t))}{t^{2}} d t .
\end{aligned}
$$

Thus

$$
\begin{gathered}
\left\|I_{2}\right\|_{X}=O(1)\left[\frac{\pi}{n+1} H\left(\frac{\pi}{n+1}\right)\right. \\
\left.+\sum_{k=0}^{\infty}\left|a_{n, k}-a_{n, k+r}\right| H\left(\frac{\pi}{n+1}\right)\right] .
\end{gathered}
$$

Collecting these estimates we obtain the first result.

Applying condition (18) we have

$$
\begin{aligned}
& {\left[(n+1) \sum_{k=0}^{\infty}\left|a_{n, k}-a_{n, k+r}\right|\right]^{-1}} \\
& =\left[\sum_{l=0}^{n} \sum_{k=0}^{\infty}\left|a_{n, k}-a_{n, k+r}\right|\right]^{-1} \\
& \leq\left[\sum_{l=0}^{n} \sum_{k=l}^{\infty}\left|a_{n, k}-a_{n, k+r}\right|\right]^{-1} \\
& \leq\left[\sum_{l=0}^{n} \mid \sum_{k=l}^{\infty}\left(a_{n, k}-a_{n, k+r}\right)\right]^{-1} \leq\left[\sum_{l=0}^{n} \sum_{k=l}^{r+l-1} a_{n, k}\right]^{-1} \\
& =O(1)
\end{aligned}
$$

and the second result also follows.

Proof of Theorem 3. Analogously, as in the proof of Theorem 2, we consider an odd $r$ and an even $r$. Then,

$$
T_{n, A} f(x)-f(x)=I_{1}(x)+I_{2}(x)
$$


or

$$
T_{n, A} f(x)-f(x)=I_{1}^{\prime}(x)+I_{2}(x)
$$

respectively, and

$$
\left\|T_{n, A} f-f\right\|_{X} \leq\left\|I_{1}\right\|_{X}+\left\|I_{1}^{\prime}\right\|_{X}+\left\|I_{2}\right\|_{X}
$$

Since $A_{n, r}=\sum_{k=0}^{\infty}\left|a_{n, k}-a_{n, k+r}\right| \leq 2$

$$
\begin{aligned}
& \left\|I_{1}\right\|_{X} \leq \frac{1}{\pi} \sum_{m=0}^{[r / 2]} \int_{2 m \pi / r}^{2 m \pi / r+\pi / r}\left\|\varphi_{X}(t)\right\|_{X}\left|\sum_{k=0}^{\infty} a_{n, k} D_{k, 1}(t)\right| d t \\
& =\frac{1}{\pi} \sum_{m=0}^{[r / 2]}\left(\int_{2 m \pi / r}^{2 m \pi / r+(1 / r) A_{n, r}}+\int_{2 m \pi / r+(1 / r) A_{n, r}}^{2 m \pi / r+\pi / r}\right) \\
& \cdot\left\|\varphi_{x}(t)\right\|_{X}\left|\sum_{k=0}^{\infty} a_{n, k} D_{k, 1}(t)\right| d t \\
& \left\|I_{2}\right\|_{X} \leq \frac{1}{\pi} \\
& \cdot \sum_{m=0}^{[r / 2]-1} \int_{2 m \pi / r+\pi / r}^{2(m+1) \pi / r}\left\|\varphi_{x}(t)\right\|_{X}\left|\sum_{k=0}^{\infty} a_{n, k} D_{k, 1}(t)\right| d t \leq \frac{1}{\pi} \\
& \quad \cdot \sum_{m=0}^{[r / 2]-1}\left(\int_{2(m+1) \pi / r-\pi / r}^{2(m+1) \pi / r-(1 / r) A_{n, r}}+\int_{2(m+1) \pi / r-(1 / r) A_{n, r}}^{2(m+1) \pi / r}\right) \\
& \cdot\left\|\varphi_{X}(t)\right\|_{X}\left|\sum_{k=0}^{\infty} a_{n, k} D_{k, 1}(t)\right| d t .
\end{aligned}
$$

Therefore, in the terms $I_{1}, I_{1}^{\prime}$, and $I_{2}$ we can estimate analogously as in the proof of Theorem 2 and thus we obtain the desired estimate.

Proof of Theorem 4. Similarly, as in the proof of Theorem 2, we consider an odd $r$ and an even $r$. Then,

$$
\begin{aligned}
T_{n, A} f(x)-f(x) & \\
= & \frac{1}{\pi} \int_{0}^{\pi / r(n+1)} \varphi_{x}(t) \sum_{k=0}^{\infty} a_{n, k} D_{k, 1}(t) d t \\
& +\frac{1}{\pi} \int_{\pi / r(n+1)}^{\pi / r} \varphi_{x}(t) \sum_{k=0}^{\infty} a_{n, k} D_{k, 1}(t) d t
\end{aligned}
$$

$$
\begin{aligned}
& +\frac{1}{\pi} \sum_{m=1}^{[r / 2]} \int_{2 m \pi / r}^{2 m \pi / r+\pi / r} \varphi_{x}(t) \sum_{k=0}^{\infty} a_{n, k} D_{k, 1}(t) d t \\
& +\frac{1}{\pi} \sum_{m=0}^{[r / 2]-1} \int_{2 m \pi / r+\pi / r}^{2(m+1) \pi / r} \varphi_{x}(t) \sum_{k=0}^{\infty} a_{n, k} D_{k, 1}(t) d t \\
& =J_{1}(x)+J_{2}(x)+I_{1}^{\prime \prime}(x)+I_{2}(x)
\end{aligned}
$$

or

$$
\begin{aligned}
& T_{n, A} f(x)-f(x)=J_{1}(x)+J_{2}(x) \\
& +\frac{1}{\pi}\left(\sum_{m=1}^{[r / 2]-1} \int_{2 m \pi / r}^{2 m \pi / r+\pi / r}+\sum_{m=0}^{[r / 2]-1} \int_{2 m \pi / r+\pi / r}^{2(m+1) \pi / r}\right) \varphi_{x}(t) \\
& \quad \cdot \sum_{k=0}^{\infty} a_{n, k} D_{k, 1}(t) d t=J_{1}(x)+J_{2}(x)+I_{1}^{\prime \prime \prime}(x) \\
& +I_{2}(x)
\end{aligned}
$$

respectively. Therefore,

$$
\begin{gathered}
\left\|T_{n, A} f-f\right\|_{X} \leq \\
+\left\|J_{1}\right\|_{X}+\left\|J_{2}\right\|_{X}+\left\|I_{1}^{\prime \prime}\right\|_{X}+\left\|I_{1}^{\prime \prime \prime}\right\|_{X} \\
+\left\|I_{2}\right\|_{X} .
\end{gathered}
$$

By Lemma 10 and (18),

$$
\begin{aligned}
\left\|J_{1}\right\|_{X} & \leq \frac{1}{\pi} \int_{0}^{\pi / r(n+1)}\left\|\varphi_{X}(t)\right\|_{X}\left|\sum_{k=0}^{\infty} a_{n, k} D_{k, 1}(t)\right| d t \\
& \leq \frac{1}{\pi} \sum_{k=0}^{\infty}(k+1) a_{n, k} \int_{0}^{\pi / r(n+1)} \omega(t) d t \\
& =O(1)(n+1) \int_{0}^{\pi / r(n+1)} \omega(t) d t \\
& \leq O(1) \frac{\pi}{r} \omega\left(\frac{\pi}{r(n+1)}\right)=O\left(\omega\left(\frac{\pi}{n+1}\right)\right) .
\end{aligned}
$$

Further, by the same lemmas and conditions as above and Lemma 15, we obtain, with

$$
\kappa= \begin{cases}1 & \text { when } r \text { is even, } \\ 0 & \text { when } r \text { is odd }\end{cases}
$$

that

$$
\begin{aligned}
\left\|I_{1}^{\prime \prime}\right\|_{X}+\left\|J_{2}\right\|_{X}+\left\|I_{1}^{\prime \prime \prime}\right\|_{X} & \leq \frac{1}{\pi}\left(\sum_{m=1}^{[r / 2]-\kappa} \int_{2 m \pi / r}^{2 m \pi / r+\pi / r}+\int_{\pi / r(n+1)}^{\pi / r}\right)\left\|\varphi_{X}(t)\right\|_{X}\left|\sum_{k=0}^{\infty} a_{n, k} D_{k, 1}(t)\right| d t \\
& =\frac{1}{\pi}\left(\sum_{m=1}^{[r / 2]-\kappa} \int_{2 m \pi / r}^{2 m \pi / r+\pi / r(n+1)}+\sum_{m=0}^{[r / 2]-\kappa} \int_{2 m \pi / r+\pi / r(n+1)}^{2 m \pi / r+\pi / r}\right)\left\|\varphi_{x}(t)\right\|_{X}\left|\sum_{k=0}^{\infty} a_{n, k} D_{k, 1}(t)\right| d t
\end{aligned}
$$




$$
\begin{aligned}
& \leq \frac{1}{\pi} \sum_{m=1}^{[r / 2]-\kappa} \int_{2 m \pi / r}^{2 m \pi / r+\pi / r(n+1)} \frac{O(\omega(t))}{2|\sin (t / 2)|} d t \\
& +\frac{1}{\pi} \sum_{m=0}^{[r / 2]-\kappa} \int_{2 m \pi / r+\pi / r(n+1)}^{2 m \pi / r+\pi / r} \frac{O(\omega(t))}{|\sin (t / 2) \sin (r t / 2)|} \sum_{k=0}^{\infty}\left|a_{n, k}-a_{n, k+r}\right| d t \\
& \leq \frac{1}{2} \sum_{m=1}^{[r / 2]-\kappa} \int_{2 m \pi / r}^{2 m \pi / r+\pi / r(n+1)} \frac{O(\omega(t))}{t} d t+\sum_{k=0}^{\infty}\left|a_{n, k}-a_{n, k+r}\right| \sum_{m=0}^{[r / 2]-\kappa} \int_{2 m \pi / r+\pi / r(n+1)}^{2 m \pi / r+\pi / r} \frac{O(\omega(t))}{t(r t / \pi-2 m)} d t \\
& \leq \sum_{m=1}^{[r / 2]-\kappa} \frac{O(\omega(2 m \pi / r))}{2 m \pi / r} \int_{2 m \pi / r}^{2 m \pi / r+\pi / r(n+1)} d t \\
& +\sum_{k=0}^{\infty}\left|a_{n, k}-a_{n, k+r}\right| \sum_{m=0}^{[r / 2]-\kappa} \int_{\pi / r(n+1)}^{\pi / r} \frac{O(\omega(t+2 m \pi / r))}{(r t / \pi)(t+2 m \pi / r)} d t \\
& \leq 2 \sum_{m=1}^{[r / 2]-\kappa} \frac{O(\omega(2 \pi / r))}{2 \pi / r} \frac{\pi}{r(n+1)}+\frac{2 \pi}{r}\left(\left[\frac{r}{2}\right]+1\right) \sum_{k=0}^{\infty}\left|a_{n, k}-a_{n, k+r}\right| \int_{\pi / r(n+1)}^{\pi / r} \frac{O(\omega(t))}{t^{2}} d t \\
& =O(1)\left[\omega\left(\frac{\pi}{n+1}\right)+\sum_{k=0}^{\infty}\left|a_{n, k}-a_{n, k+r}\right| H\left(\frac{\pi}{n+1}\right)\right] \text {, } \\
& \left\|I_{2}\right\|_{X} \leq \frac{1}{\pi} \sum_{m=0}^{[r / 2]-1}\left(\int_{2(m+1) \pi / r-\pi / r}^{2(m+1) \pi / r-\pi / r(n+1)}+\int_{2(m+1) \pi / r-\pi / r(n+1)}^{2(m+1) \pi / r}\right)\left\|\varphi_{X}(t)\right\|_{X}\left|\sum_{k=0}^{\infty} a_{n, k} D_{k, 1}(t)\right| d t \\
& \leq \frac{1}{\pi} \sum_{m=0}^{[r / 2]-1} \int_{2(m+1) \pi / r-\pi / r}^{2(m+1) \pi / r-\pi / r(n+1)} \frac{O(\omega(t))}{|\sin (t / 2) \sin (r t / 2)|} \sum_{k=0}^{\infty}\left|a_{n, k}-a_{n, k+r}\right| d t \\
& +\frac{1}{2} \sum_{m=0}^{[r / 2]-1} \int_{2(m+1) \pi / r-\pi / r(n+1)}^{2(m+1) \pi / r} \frac{O(\omega(t))}{t} d t \\
& \leq \sum_{m=0}^{[r / 2]-1} \sum_{k=0}^{\infty}\left|a_{n, k}-a_{n, k+r}\right| \int_{2(m+1) \pi / r-\pi / r}^{2(m+1) \pi / r-\pi / r(n+1)} \frac{O(\omega(t))}{(r t / \pi)[2(m+1) \pi / r-t]} d t \\
& +\sum_{m=0}^{[r / 2]-1} \frac{O(\omega(2(m+1) \pi / r-\pi / r(n+1)))}{2(m+1) \pi / r-\pi / r(n+1)} \int_{2(m+1) \pi / r-\pi / r(n+1)}^{2(m+1) \pi / r} d t \\
& \leq\left[\frac{r}{2}\right]\left[\frac{2 \pi}{r} \sum_{k=0}^{\infty}\left|a_{n, k}-a_{n, k+r}\right| \int_{\pi / r(n+1)}^{\pi / r} \frac{O(\omega(t))}{t^{2}} d t+2 \frac{O(\omega(\pi / r))}{\pi / r} \frac{\pi}{r(n+1)}\right] \\
& =O(1)\left[\sum_{k=0}^{\infty}\left|a_{n, k}-a_{n, k+r}\right| H\left(\frac{\pi}{n+1}\right)+\omega\left(\frac{\pi}{n+1}\right)\right] \text {. }
\end{aligned}
$$

Thus our proof is complete.

$$
\left\|J_{1}\right\|_{X} \leq \frac{1}{\pi} \int_{0}^{\pi / r(n+1)}\left\|\varphi_{x}(t)\right\|_{X}\left|\sum_{\mathrm{k}=0}^{\infty} a_{n, k} D_{k, 1}(t)\right| d t
$$

Proof of Theorem 5. Let as above

$$
\begin{aligned}
& \leq \frac{1}{\pi} \sum_{k=0}^{\infty}(k+1) a_{n, k} \int_{0}^{\pi / r(n+1)} \omega_{2}(f, t)_{X} d t \\
& =O(n+1) \int_{0}^{\pi / r(n+1)} \omega_{2}(f, t)_{X} d t
\end{aligned}
$$$$
\left\|T_{n, A} f-f\right\|_{X}
$$$$
\leq\left\|J_{1}\right\|_{X}+\left\|J_{2}\right\|_{X}+\left\|I_{1}^{\prime \prime}\right\|_{X}+\left\|I_{1}^{\prime \prime \prime}\right\|_{X}+\left\|I_{2}\right\|_{X},
$$ 


$$
\begin{aligned}
& \leq O(1) \frac{\pi}{r} \omega_{2}\left(f, \frac{\pi}{r(n+1)}\right)_{X} \\
& =O(1) \omega_{2}\left(f, \frac{\pi}{n+1}\right)_{X} .
\end{aligned}
$$

Further, taking $\tau_{m}^{1}=[\pi /(r t-2 m \pi)]$ and $\tau=[\pi / r t]$, using Lemma 15, we obtain, with

$$
\kappa= \begin{cases}1 \quad \text { when } r \text { is even, } \\ 0 \quad \text { when } r \text { is odd }\end{cases}
$$

that

$$
\begin{aligned}
\left\|I_{1}^{\prime \prime}\right\|_{X}+\left\|J_{2}\right\|_{X}+\left\|I_{1}^{\prime \prime \prime}\right\|_{X} \leq \frac{1}{\pi}\left(\sum_{m=1}^{[r / 2]-\kappa} \int_{2 m \pi / r}^{2 m \pi / r+\pi / r}\right. \\
\left.+\int_{\pi / r(n+1)}^{\pi / r}\right)\left\|\varphi_{x}(t)\right\|_{X}\left|\sum_{k=0}^{\infty} a_{n, k} D_{k, 1}(t)\right| d t \\
=\frac{1}{\pi}\left(\sum_{m=1}^{[r / 2]-\kappa} \int_{2 m \pi / r}^{2 m \pi / r+\pi / r(n+1)}\right. \\
\left.+\sum_{m=0}^{[r / 2]-\kappa} \int_{2 m \pi / r+\pi / r(n+1)}^{2 m \pi / r+\pi / r}\right)\left\|\varphi_{x}(t)\right\|_{X} \\
.\left|\sum_{k=0}^{\infty} a_{n, k} D_{k, 1}(t)\right| d t \leq \frac{1}{\pi} \mid \frac{\omega_{2}(f, t)_{X}}{2|\sin (t / 2)|} \sum_{k=0}^{\infty} a_{n, k} d t+\frac{1}{\pi} \\
\quad \cdot \sum_{m=1}^{[r / 2]-\kappa} \int_{2 m \pi / r}^{2 m \pi / r+\pi / r(n+1)} \\
\quad \sum_{m=0}^{[r / 2]-\kappa} \int_{2 m \pi / r+\pi / r(n+1)}^{2 m \pi / r+\pi / r}\left(\frac{\omega_{2}(f, t)_{X}}{2|\sin (t / 2)|} \sum_{k=0}^{\tau_{m}^{1}} a_{n, k}\right.
\end{aligned}
$$

$$
\begin{aligned}
\left\|I_{2}\right\|_{X} \leq & \frac{1}{\pi} \sum_{m=0}^{[r / 2]-1} \int_{2 m \pi / r+\pi / r}^{2(m+1) \pi / r}\left\|\varphi_{X}(t)\right\|_{X}\left|\sum_{k=0}^{\infty} a_{n, k} D_{k, 1}(t)\right| d t \\
\leq & \frac{1}{\pi} \sum_{m=0}^{[r / 2]-1}\left(\int_{2(m+1) \pi / r-\pi / r}^{2(m+1) \pi / r-\pi / r(n+1)}+\int_{2(m+1) \pi / r-\pi / r(n+1)}^{2(m+1) \pi / r}\right)\left\|\varphi_{X}(t)\right\|_{X}\left|\sum_{k=0}^{\infty} a_{n, k} D_{k, 1}(t)\right| d t \\
\leq & \frac{1}{\pi} \sum_{m=0}^{[r / 2]-1} \int_{2(m+1) \pi / r-\pi / r}^{2(m+1) \pi / r-\pi / r(n+1)}\left(\frac{\omega_{2}(f, t)_{X}}{2|\sin (t / 2)|} \sum_{k=0}^{\tau_{m}^{2}} a_{n, k}+\frac{\omega_{2}(f, t)_{X}}{|\sin (t / 2) \sin (r t / 2)|} \sum_{k=\tau_{m}^{2}}^{\infty}\left|a_{n, k}-a_{n, k+r}\right|\right) d t \\
& +\frac{1}{\pi} \sum_{m=0}^{[r / 2]-1} \int_{2(m+1) \pi / r-\pi / r(n+1)}^{2(m+1) \pi / r} \frac{\omega_{2}(f, t)_{X}}{2|\sin (t / 2)|} \sum_{k=0}^{\infty} a_{n, k} d t \\
\leq & \frac{1}{2} \sum_{m=0}^{[r / 2]-1} \int_{\pi / r(n+1)}^{\pi / r} \frac{\omega_{2}(-t+2(m+1) \pi / r)_{X}}{-t+2(m+1) \pi / r} \sum_{k=0}^{\tau} a_{n, k} d t
\end{aligned}
$$

Next, taking $\tau_{m}^{2}=[\pi /(-r t+2(m+1) \pi)]$, we obtain
$+1) \int_{\pi / r(n+1)}^{\pi / r} \frac{\omega_{2}(f, t)_{X}}{t} \sum_{k=0}^{\tau} a_{n, k} d t+\frac{2 \pi}{r}\left(\left[\frac{r}{2}\right]+1\right)$

$\cdot \int_{\pi / r(n+1)}^{\pi / r} \frac{\omega_{2}(f, t)_{X}}{t^{2}} \sum_{k=\tau}^{\infty}\left|a_{n, k}-a_{n, k+r}\right| d t$

$\leq \sum_{m=1}^{[r / 2]-\kappa} \frac{\omega_{2}(f, 2 m \pi / r)_{X}}{2 m \pi / r} \int_{2 m \pi / r}^{2 m \pi / r+\pi / r(n+1)} d t$

$+\frac{1}{2}\left(\left[\frac{r}{2}\right]+1\right) \sum_{\mu=1}^{n} \int_{\mu}^{\mu+1} \frac{\omega_{2}(f, \pi / r t)_{X}}{\pi / r t} \sum_{k=0}^{[t]} a_{n, k} \frac{\pi d t}{r t^{2}}$

$+\frac{2 \pi}{r}\left(\left[\frac{r}{2}\right]+1\right)$

$\cdot \sum_{\mu=1}^{n} \int_{\mu}^{\mu+1} \frac{\omega_{2}(f, \pi / r t)_{X}}{(\pi / r t)^{2}} \sum_{k=[t]}^{\infty}\left|a_{n, k}-a_{n, k+r}\right| \frac{\pi d t}{r t^{2}}$

$\leq O(1) \omega_{2}\left(f, \frac{\pi}{n+1}\right)_{X}+O(1)$

$\cdot \sum_{\mu=1}^{n} \frac{\omega_{2}(f, \pi / \mu)_{X}}{\mu} \sum_{k=0}^{\mu+1} a_{n, k}+O(1) \sum_{\mu=1}^{n} \omega_{2}\left(f, \frac{\pi}{\mu}\right)_{X}$

$\sum_{k=\mu}^{\infty}\left|a_{n, k}-a_{n, k+r}\right|$ 


$$
\begin{aligned}
& +\sum_{m=0}^{[r / 2]-1} \int_{\pi / r(n+1)}^{\pi / r} \frac{\omega_{2}(-t+2(m+1) \pi / r)_{X}}{(r / \pi) t(-t+2(m+1) \pi / r)} \sum_{k=\tau}^{\infty}\left|a_{n, k}-a_{n, k+r}\right| d t+\frac{1}{2} \sum_{m=0}^{[r / 2]-1} \int_{2(m+1) \pi / r-\pi / r(n+1)}^{2(m+1) \pi / r} \frac{\omega_{2}(t)_{X}}{t} d t \\
\leq & {\left[\frac{r}{2}\right] \int_{\pi / r(n+1)}^{\pi / r} \frac{\omega_{2}(f, t)_{X}}{t} \sum_{k=0}^{\tau} a_{n, k}+\frac{2 \pi}{r}\left[\frac{r}{2}\right] \int_{\pi / r(n+1)}^{\pi / r} \frac{\omega_{2}(f, t)_{X}}{t^{2}} \sum_{k=\tau}^{\infty}\left|a_{n, k}-a_{n, k+r}\right| d t } \\
& +\sum_{m=0}^{[r / 2]-1} \frac{\omega_{2}(f, 2(m+1) \pi / r-\pi / r(n+1))_{X}}{2(m+1) \pi / r-\pi / r(n+1)} \int_{2(m+1) \pi / r-\pi / r(n+1)}^{2(m+1) \pi / r} d t \\
\leq & O(1) \sum_{\mu=1}^{n} \frac{\omega_{2}(f, \pi / \mu)_{X}}{\mu} \sum_{k=0}^{\mu+1} a_{n, k}+O(1) \sum_{\mu=1}^{n} \omega_{2}\left(f, \frac{\pi}{\mu}\right)_{X} \sum_{k=\mu}^{\infty}\left|a_{n, k}-a_{n, k+r}\right|+O(1) \omega_{2}\left(f, \frac{\pi}{n+1}\right)_{X} .
\end{aligned}
$$

Thus the result follows.

Proof of Corollary 7. Theorem 5 implies that

$$
\begin{aligned}
& \left\|T_{n, A} f-f\right\|_{X}=O\left(\omega_{2}\left(f, \frac{\pi}{n+1}\right)_{X}\right. \\
& +\sum_{\mu=1}^{n} \frac{\omega_{2}(f, \pi / \mu)_{X}}{\mu} \sum_{k=0}^{\mu+1} a_{n, k} \\
& \left.+\sum_{\mu=1}^{n} \omega_{2}\left(f, \frac{\pi}{\mu}\right)_{X} \sum_{k=\mu}^{\infty}\left|a_{n, k}-a_{n, k+r}\right|\right) .
\end{aligned}
$$

Since in (24)

$$
\begin{aligned}
& \sum_{\mu=1}^{n} \omega_{2}\left(f, \frac{\pi}{\mu}\right)_{X} \sum_{k=\mu}^{\infty}\left|a_{n, k}-a_{n, k+r}\right|=O(1) \\
& \cdot \sum_{\mu=1}^{n} \omega_{2}\left(f, \frac{\pi}{\mu}\right)_{X}\left(\sum_{k=\mu / c}^{\mu-1} \frac{a_{n, k}}{k}+\sum_{k=\mu}^{n} \frac{a_{n, k}}{k}+\sum_{k=n+1}^{\infty} \frac{a_{n, k}}{k}\right) \\
& \leq O(1) \sum_{\mu=1}^{n} \omega_{2}\left(f, \frac{\pi}{\mu}\right)_{X}\left(\sum_{k=\mu / c}^{\mu-1} \frac{a_{n, k}}{k}\right)+O(1) \\
& \cdot \sum_{\mu=1}^{n} \omega_{2}\left(f, \frac{\pi}{\mu}\right)_{X}\left(\sum_{k=\mu}^{n} \frac{a_{n, k}}{k}+\sum_{k=n+1}^{\infty} \frac{a_{n, k}}{k}\right) \leq O(1) \\
& \cdot c \sum_{\mu=1}^{n} \frac{\omega_{2}(f, \pi / \mu)_{X}}{\mu+1} \sum_{k=0}^{\mu+1} a_{n, k}+O(1) \sum_{\mu=1}^{n} \omega_{2}\left(f, \frac{\pi}{\mu}\right)_{X} \\
& \cdot\left(\sum_{k=\mu}^{n} \frac{a_{n, k}}{k}+\sum_{k=n+1}^{\infty} \frac{a_{n, k}}{k}\right)
\end{aligned}
$$

one has

$$
\left\|T_{n, A} f-f\right\|_{X}=O(1) \omega_{2}\left(f, \frac{\pi}{n+1}\right)_{X}+O(1)(1+c)
$$

$\cdot \sum_{\mu=1}^{n} \frac{\omega_{2}(f, \pi / \mu)_{X}}{\mu} \sum_{k=0}^{\mu+1} a_{n, k}+O(1)$

$\cdot \sum_{\mu=1}^{n} \omega_{2}\left(f, \frac{\pi}{\mu}\right)_{X} \sum_{k=\mu}^{n} \frac{a_{n, k}}{k}+O(1)$

$\cdot \sum_{k=n+1}^{\infty} \frac{a_{n, k}}{k} \sum_{\mu=1}^{n} \omega_{2}\left(f, \frac{\pi}{\mu}\right)_{X} \leq O(1) \omega_{2}\left(f, \frac{\pi}{n+1}\right)_{X}$

$+O(1)(1+c)\left\{\sum_{\mu=1}^{n} \frac{\omega_{2}(f, \pi / \mu)_{X}}{\mu-1} \sum_{k=0}^{\mu-1} a_{n, k}\right.$

$\left.+2 \sum_{\mu=1}^{n} \frac{\omega_{2}(f, \pi / \mu)_{X}}{\mu+1} a_{n, \mu+1}+\sum_{\mu=1}^{n} \frac{\omega_{2}(f, \pi / \mu)_{X}}{\mu} a_{n, \mu}\right\}$

$+O(1) \sum_{\mu=1}^{n} \omega_{2}\left(f, \frac{\pi}{\mu}\right) \sum_{X=\mu}^{n} \frac{a_{n, k}}{k}+O(1)$

$\cdot \sum_{k=n+1}^{\infty} \frac{a_{n, k}}{k} \sum_{\mu=1}^{n} \omega_{2}\left(f, \frac{\pi}{\mu}\right)_{X} \leq O(1) \omega_{2}\left(f, \frac{\pi}{n+1}\right)_{X}$

$+O(1)(1+c) \sum_{\mu=0}^{n} \frac{\omega_{2}(f, \pi /(\mu+1))_{X}}{\mu+1} \sum_{k=0}^{\mu} a_{n, k}$

$+O(1)[3(1+c)+1] \sum_{\mu=1}^{n} \omega_{2}\left(f, \frac{\pi}{\mu}\right)_{X} \sum_{k=\mu}^{n} \frac{a_{n, k}}{k}$

$+O(1) \sum_{k=n+1}^{\infty} \frac{a_{n, k}}{k} \sum_{\mu=1}^{n} \omega_{2}\left(f, \frac{\pi}{\mu}\right)_{X}=O(1)$

$\cdot \omega_{2}\left(f, \frac{\pi}{n+1}\right)_{X}+O(1)$

$\cdot \sum_{k=0}^{n} a_{n, k} \sum_{\mu=k}^{n} \frac{\omega_{2}(f, \pi /(\mu+1))_{X}}{\mu+1}+O(1)$

$\cdot \sum_{k=1}^{n} \frac{a_{n, k}}{k} \sum_{\mu=1}^{k} \omega_{2}\left(f, \frac{\pi}{\mu}\right)_{X}+O(1)$ 


$$
\cdot \sum_{k=n+1}^{\infty} \frac{a_{n, k}}{k} \sum_{\mu=1}^{n} \omega_{2}\left(f, \frac{\pi}{\mu}\right)_{X}
$$

If (14) and (15) hold, then

$$
\begin{aligned}
& \omega_{2}\left(f, \frac{\pi}{n+1}\right)_{X} \leq \frac{1}{n} \sum_{\mu=1}^{n} \omega_{2}\left(f, \frac{\pi}{\mu}\right)_{X} \\
& \leq O(1) 4 \pi \frac{H(\pi /(n+1))}{n+1}, \\
& \sum_{\mu=k}^{n} \frac{\omega_{2}(f, \pi /(\mu+1))_{X}}{\mu+1} \leq 8 \int_{\pi /(n+2)}^{\pi /(k+1)} \frac{\omega_{2}(f, t)_{X}}{t} d t \\
& \leq O \text { (1) } 8 \pi \frac{H(\pi /(k+1))}{k+1}
\end{aligned}
$$

and therefore

$$
\begin{aligned}
\left\|T_{n, A} f-f\right\|_{X}= & O\left(\frac{H(\pi /(n+1))}{n+1}\right) \\
& +O\left(\sum_{k=1}^{n} a_{n, k} \frac{H(\pi /(k+1))}{k+1}\right) \\
& +O\left(H\left(\frac{\pi}{n+1}\right) \sum_{k=n+1}^{\infty} \frac{a_{n, k}}{k}\right) .
\end{aligned}
$$

Since

$$
\sum_{k=n+1}^{\infty} \frac{a_{n, k}}{k} \leq \frac{1}{n+1} \sum_{k=n+1}^{\infty} a_{n, k}=O\left(\frac{1}{n+1}\right)
$$

the result follows (cf. [6]).

\section{Competing Interests}

The authors declare that they have no competing interests.

\section{Acknowledgments}

The authors would like to express their thanks to Professor S. Tikhonov for valuable suggestions concerning Corollary 7 and to referee for the information concerning Remark 9.

\section{References}

[1] X. Z. Krasniqi, "Some further results on the degree of approximation of continuous functions," Annales Universitatis Scientiarum Budapestinensis de Rolando Eötvös Nominatae. Sectio Computatorica, vol. 38, pp. 279-294, 2012.

[2] B. Wei and D. Yu, "On the degree of approximation of continuous functions by means of Fourier series," Mathematical Communications, vol. 17, no. 1, pp. 211-219, 2012.

[3] B. Szal, "A new class of numerical sequences and its applications to uniform convergence of sine series," Mathematische Nachrichten, vol. 284, no. 14-15, pp. 1985-2002, 2011.
[4] B. Szal, "On weighted $L^{p}$ integrability of functions defined by trigonometric series," Journal of Inequalities and Applications, vol. 2010, Article ID 485705, 19 pages, 2010.

[5] M. Dyachenko and S. Tikhonov, "Integrability and continuity of functions represented by trigonometric series: coefficients criteria," Studia Mathematica, vol. 193, no. 3, pp. 285-306, 2009.

[6] S. Tikhonov, "Trigonometric series with general monotone coefficients," Journal of Mathematical Analysis and Applications, vol. 326, no. 1, pp. 721-735, 2007.

[7] B. Szal, "On L-convergence of trigonometric series," Journal of Mathematical Analysis and Applications, vol. 373, no. 2, pp. 449463, 2011.

[8] A. Zygmund, Trigonometric Series, Cambridge University Press, Cambridge, UK, 2002.

[9] P. Chandra, "A note on the degree of approximation of continuous functions," Acta Mathematica Hungarica, vol. 62, no. 1-2, pp. 21-23, 1993. 


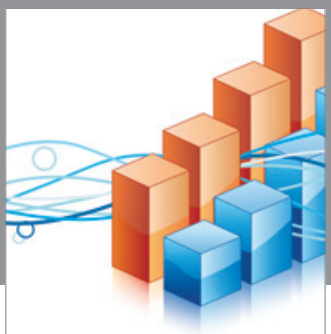

Advances in

Operations Research

vatem alat4

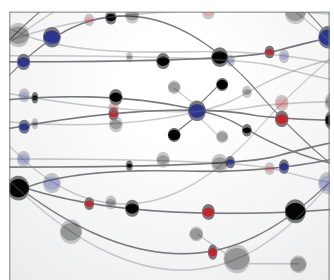

\section{The Scientific} World Journal
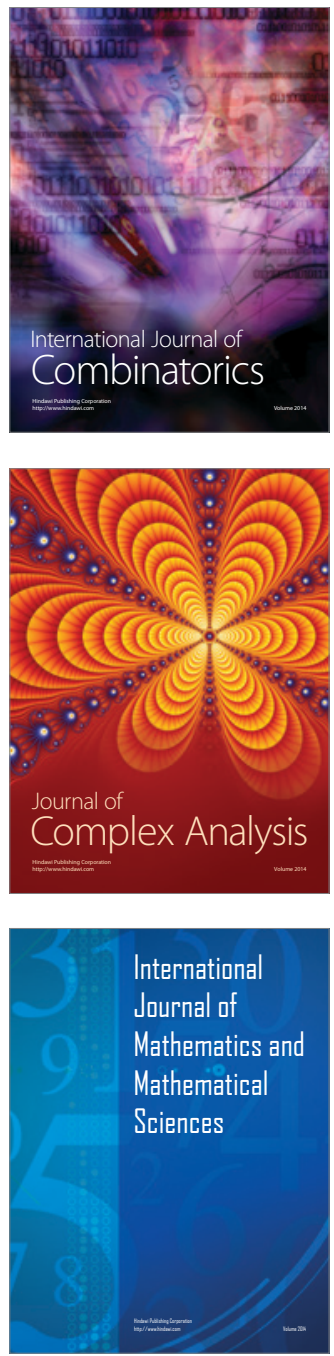
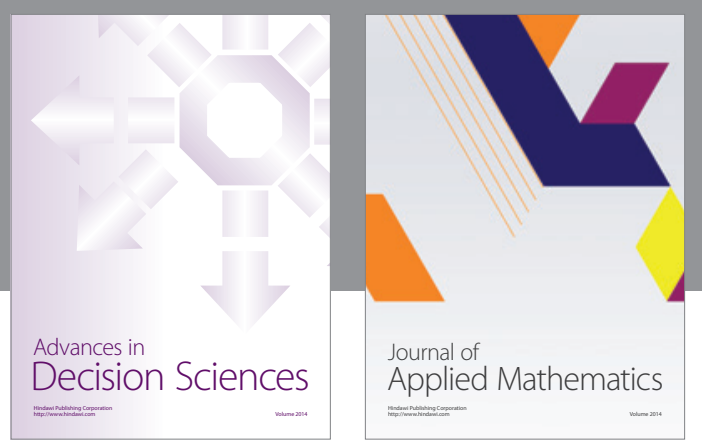

Algebra

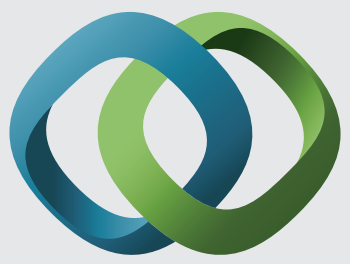

\section{Hindawi}

Submit your manuscripts at

http://www.hindawi.com
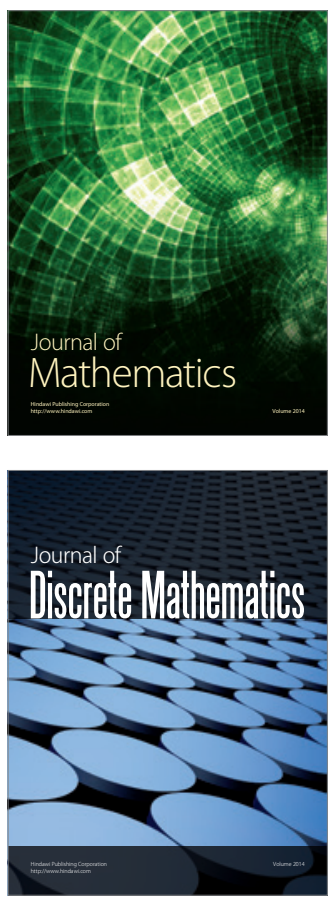

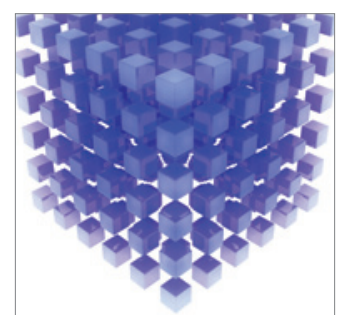

Mathematical Problems in Engineering
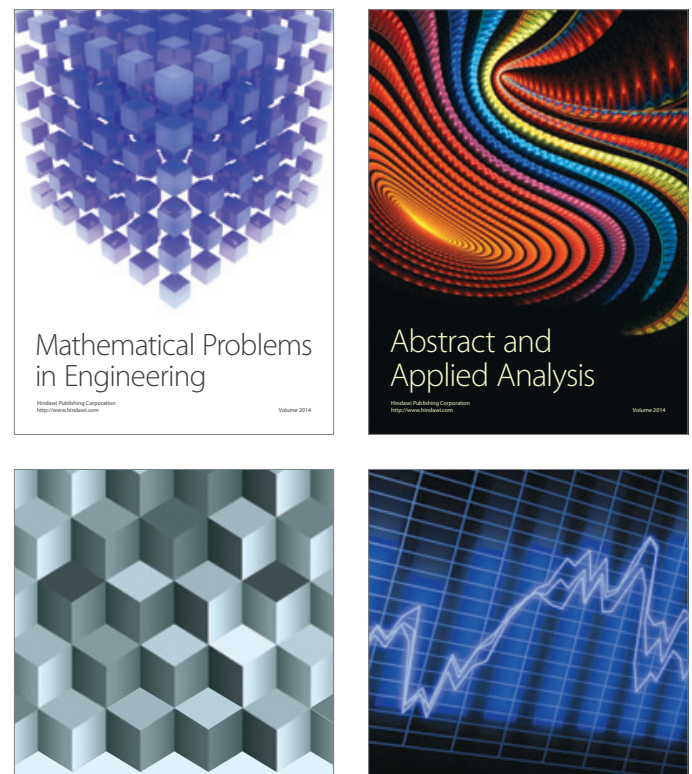

Journal of

Function Spaces

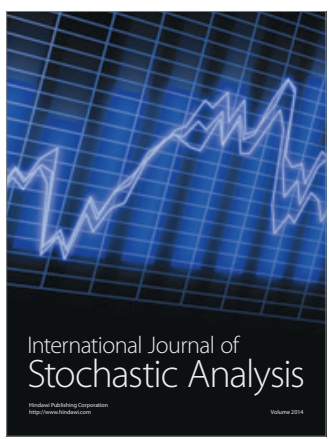

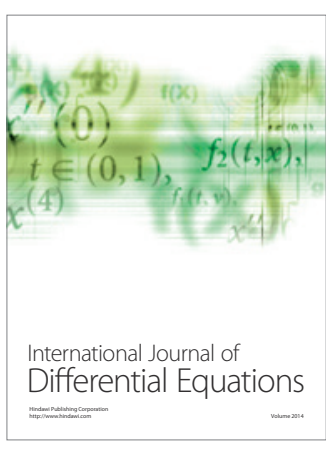
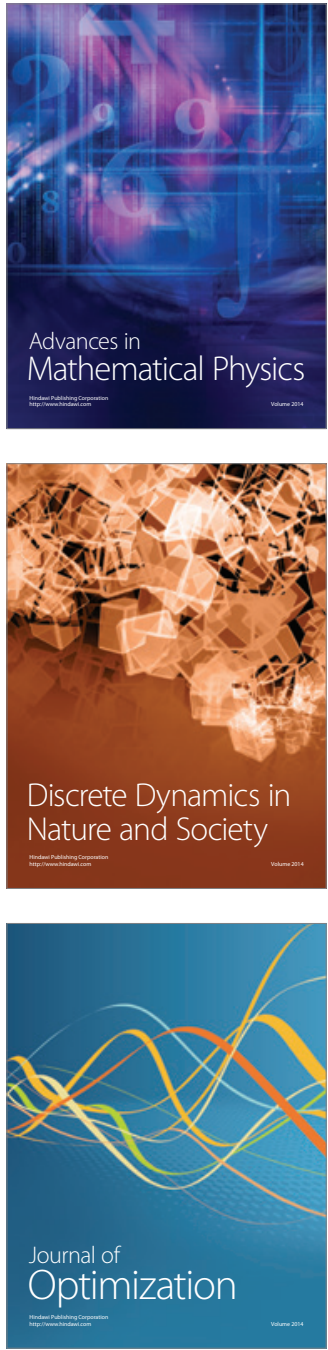